\title{
Using Haptic Communications with the Leg to Maintain Exercise Intensity
}

\author{
Aaron R. Ferber, Michael Peshkin, Member, IEEE, J. Edward Colgate, Member, IEEE \\ Mechanical Engineering, Northwestern University, Evanston, IL, USA \\ \{aaronferber, peshkin, colgate\}@ northwestern.edu
}

\begin{abstract}
The haptic sense provides continuous information during physical human-machine interaction. Humans can respond very quickly and effectively to such feedback, such as a driver making rapid steering adjustments when his vehicle hits a pothole. It may be possible for designers to take advantage of this interaction by providing users with welldesigned haptic communications to assist in manual control tasks. In this paper, we describe an experiment conducted to test this idea. Subjects were instructed to step on a modified stair climber at a consistent workout pace while simultaneously completing a mental distraction task. They were provided with one of five different haptic communications to assist in maintaining their workout intensity level. Results show that haptic communications helped subjects step as much as $1.7 \%$ faster with as much as $3.95 \%$ more power output than without the haptic communications, although none of the communications beneficially affected velocity or power standard deviations. Four of the five communications had no significant impact on mental task performance.
\end{abstract}

\section{INTRODUCTION}

Haptic interaction is a very natural method for communicating sensing and control information between a machine and its user. For example, an automobile driver can very quickly recognize that his vehicle is sliding on ice because the dynamics of the steering wheel and pedals change. However, designers have historically relied on visual and auditory channels for communicating information to a user. Machines from airplanes to exercise equipment all have an array of lights and sounds to notify the user of the system state and send warning messages. However, in the manual control of machines, the haptic channel may prove to be more effective at changing using behavior because of this natural interaction, and it may reduce the mental demand of the user as well since it spreads sensory information over more channels. In order to design these systems, we must better understand the characteristics that make haptic communications effective and unobtrusive.

\section{BACKGROUND}

The study of haptic communications spans a wide range of academic fields including human-computer interaction, product design, stroke rehabilitation, and psychology, to name a few. Much of the literature does not explicitly consider haptic communications in a manual control task, but the lessons learned in other settings are certainly relevant.

The study of haptics communications is most prevalent in the field of computer interaction. Researchers have investigated using vibrating mice [1], [2], force-feedback styluses [3], [4], [5], tactile foot platforms [6], and other haptic devices to provide feedback for tasks such as desktop navigation [2], shared control of an application [3], [7], [8], or even social interaction [9], [10], [11]. These studies have shown that individuals can successfully discriminate between different values of vibration frequency [12], location of vibration sensation [6], rhythm [13], and multiple other factors that can define haptic communications. Several studies have shown that subjects can complete these haptic discrimination tasks even in the presence of mental distractions [1], [2], [12].

Commercial entities have shown interest in haptic communications including research related to mobile devices and automobiles. These studies have shown that haptic communications can be learned and recalled in a reasonable amount of time so as to be implemented in products [14], [15]. They also show the variety of situations in which haptic communications can be used. Within the context of mobile devices, haptics have been used to notify users of information such as message type and call priority [13]. Automotive studies have shown that haptic sensations can communicate information such as navigational directions [15] and collision avoidance warnings [16], [17], [18].

There has been some work on the idea of haptic information flow between user and machine during shared control of a manual control task, most often studied within the realm of driving an automobile. These studies include haptic information received through the steering wheel [17], [19], [20] and through the pedals [21], [22]. These works have shown that providing a driver with haptic information by directly manipulating the dynamics of the control mechanisms can improve task performance while reducing visual and mental load.

We have found no previous work on the specific topic of using haptic communications in the operation of an exercise machine. There are multiple methods developed by manufacturers to assist a user in maintaining workout intensity, however. These include integrating video games [23], [24], providing synchronized music [25], providing "motivational interfaces" such as red-yellow-green light systems [26], or rewarding the user by keeping a television on if a certain power exertion level is maintained [27], [28].

This paper is taken from a thesis [29] which includes many more resources relevant to this study.

\section{EXPERIMENT OVERVIEW}

We desired an experiment that would provide a situation in which a subject was given both a physical task and a mental task that would distract the subject from the physical task. This is similar to a situation such as driving wherein a driver is physically controlling the vehicle but is mentally preoccupied with conversation or listening to the radio. The situation we designed was to give subjects the physical task of stepping at a workout pace as consistently as possible and 
the mental task of playing a video game. During part of the time the subject was doing this multitasking, we provided a haptic communication that assisted the subject in keeping a consistent pace. We then quantitatively examined whether or not the subject performed better at the physical task with the assistance of the haptic communication, and whether or not the presence of this communication affected performance on the mental task of the video game. Subjects also completed subjective questionnaires, providing us with a qualitative evaluation of each haptic communication.

\section{EXPERIMENTAL SETUP}

The stair climber: The experimental setup consisted of a modified LifeFitness 9500HR stair climber, shown in Fig. 1. We modified the stair climber such that the pedals were coupled and driven by a $1.8 \mathrm{~kW}$ Kollmorgen Goldline motor (max speed $2800 \mathrm{rpm}$, peak torque $12 \mathrm{~N} \cdot \mathrm{m}$ ). We placed four load cells underneath each foot pedal, allowing for force resolution of $\pm 0.5 \mathrm{~N}$. This force signal was used by the computer to move the pedals according to a dynamic model. We also added multiple safety switches, including limit switches underneath each pedal and ribbon switches along each handrail that could be activated by the user. Finally, we mounted a cell phone vibration motor onto each foot pedal.

Dynamic model: With the stair climber pedals controlled by a motor, it is possible to present any desired interaction to the user. We call the program defining this interaction a "dynamic model". For our experiment, we used the dynamic model of a flywheel. That is, the pedals moved as if they were connected to a flywheel, as shown in Fig. 2. This provided a very natural interaction to the user since it operated according to laws of the physical world. The inertia of the flywheel, $I$, provided the user with the momentum that is experienced when actually climbing stairs. Resistance

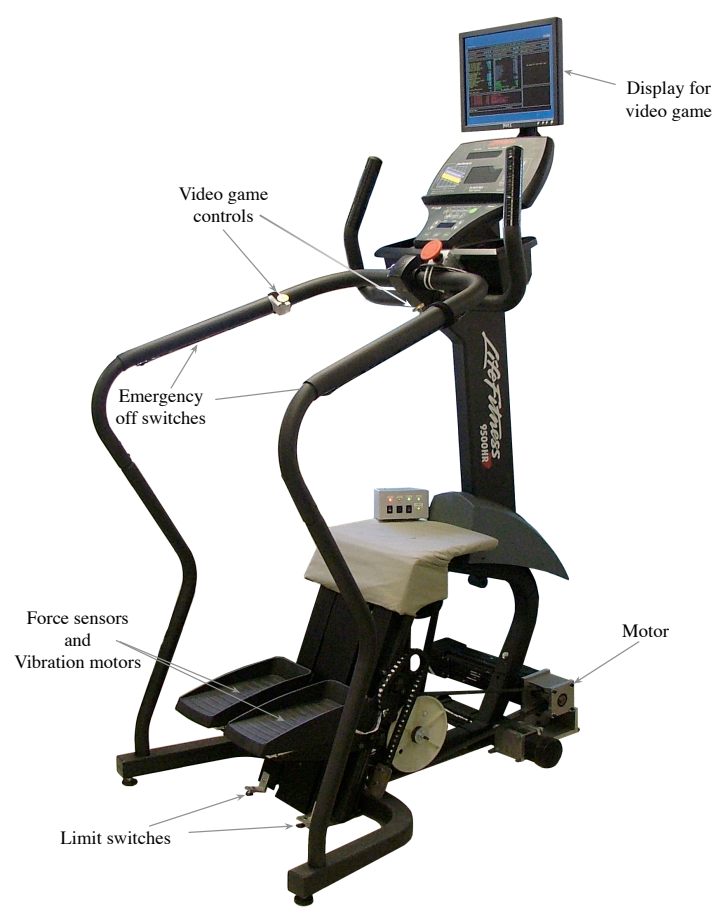

Fig. 1. Experimental stair climber

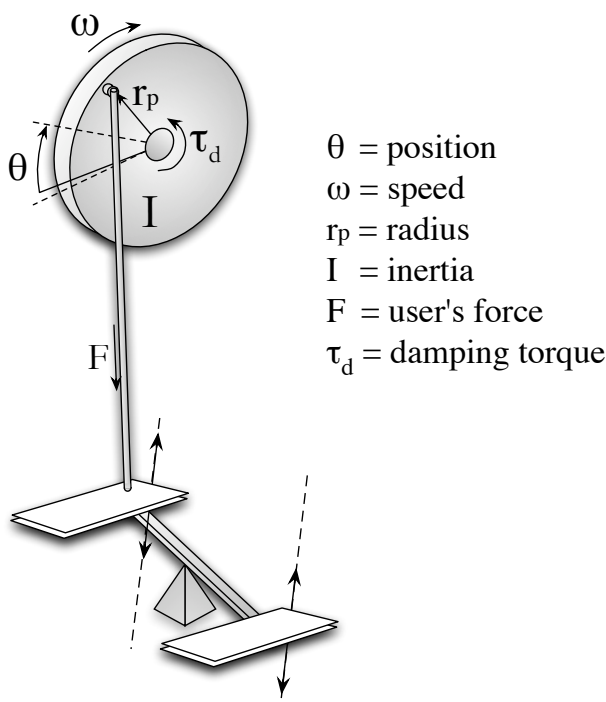

Fig. 2. The flywheel dynamic model

to user motion was regulated by adjusting the damping constant, $b$, of the damping torque, $\tau_{d}=\omega \cdot b$, exerted on the flywheel. In this experiment, we subjectively set these parameters at $I=6.0 \mathrm{~kg} \cdot \mathrm{m}^{2}$ and $b=2.0 \mathrm{~N} \cdot \mathrm{m} \cdot \mathrm{s}$. A flywheel radius, $r_{p}$, of $0.1 \mathrm{~m}$ provided a total step height of $0.2 \mathrm{~m}$.

The video game: We set up the stair climber to allow the user to play a video game while stepping. This included mounting a 15" display at eye level and mounting a knob connected to a potentiometer along each handrail. These knobs could be turned by the user's thumbs and served as the inputs for the video game. The video game we developed was similar to the game Pong ${ }^{\mathrm{TM}}$. A screenshot can be seen in Fig. 3. In this game, the user had one set of horizontal paddles and one set of vertical paddles that could be moved as depicted in Fig. 3. The objective was to use the paddles to keep the "ball" from going out of bounds. The game kept track of how many "misses" the subject had, providing a simple metric for performance on the mental task.

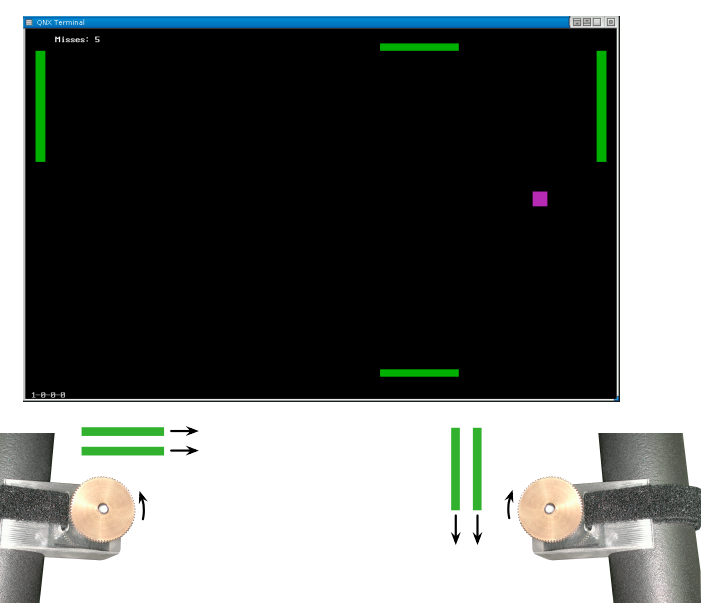

Fig. 3. Video game display and controls 


\section{HAPTIC COMMUNICATIONS}

We developed a library of haptic communications to convey information to the stair climber user and selected the following five communications to test experimentally. The purpose of each of these communications is to assist the user in the physical task of maintaining a consistent step speed of $\omega_{\text {goal }}$. For brevity, we call these "haptic cues".

Vibration: If the user steps at a pace slower than $\omega_{\text {goal }}$, the cell phone vibration motors on each pedal turn on. We subjectively set the intensity of this motor vibration. Once the user started stepping at $\omega_{\text {goal }}$ or faster, the vibration went away.

Tapping at $2 \times \omega_{\text {goal }}$ : In this model, the user can feel a tapping on his or her feet. These taps are achieved by adding a quick, brief acceleration to the motor command. The tapping occurs at a frequency such that the user would feel two taps per step if stepping at $\omega_{\text {goal }}$, but continues at this frequency regardless of the user's speed. The idea is that the user should step in sync with the beat - a haptic metronome of sorts. Two taps per step are used instead of only one tap per step at the suggestion of a pilot study subject who said it was challenging to mentally separate the haptic taps from his stepping cadence when only one tap per step was used.

E-Boost: If a user's velocity is less than $\omega_{\text {goal }}$, this model will speed up the user a small amount; we say it gives the user an "energy boost". This boost is introduced sinusoidally so as to avoid jolting the user, and it only boosts the user a small amount. That is, it does not necessarily boost the user all the way up to $\omega_{\text {goal }}$ in one boost. Boosts cannot occur back-to-back; rather, there is a set time delay between boosts. This was all done because the purpose is to tell the user to speed up, not to automatically increase his speed. An analogy to the feel of this cue is a person giving a runner a small push from behind.

Asymmetric Resistance: If the user starts stepping slower than $\omega_{\text {goal }}$, the resistance experienced on one leg increases and resistance on the other leg decreases. Thus, in theory, the user feels a discrepancy between the force required of each leg if she is not stepping at $\omega_{\text {goal }}$. This model was motivated by the work of Simon et al. [30]. In this work, the authors used a computer-controlled leg press to increase the resistance to motion experienced by a user proportionately to the asymmetry in the force the user applied. They found that users successfully adjusted the symmetry of their force in order to minimize the resistance to motion experienced. We hypothesized that if subjects of their experiment could control leg force asymmetry in this way, perhaps they could also detect an asymmetry in leg force and respond to it.

Fast Tapping: This model also creates a tapping on the user's feet, but the purpose is not to serve as a metronome (as is the case for Tapping at $2 \times \omega_{\text {goal }}$.) Rather, if the user steps slower than $\omega_{\text {goal }}$, tapping is felt at a rate of $\sim 4$ Hz. Once the user starts stepping at $\omega_{\text {goal }}$ or faster, the tapping stops.

The Asymmetric Resistance and E-Boost cues could be considered "kinesthetic" - haptic cues that are perceived globally by the user. The vibration and tapping cues are

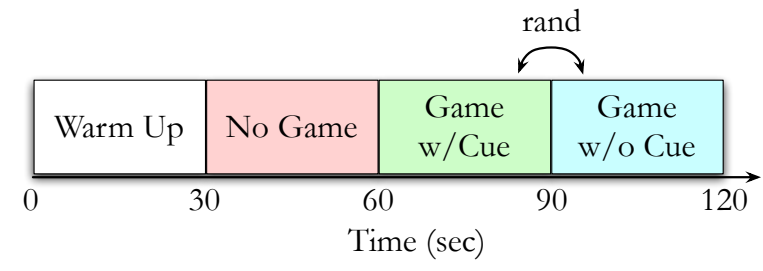

Fig. 4. Experiment timeline

more "tactile" in nature - primarily felt locally at the foot. The cues could also be classified according to methodology: Asymmetric Resistance and E-Boost both rely on an almost subconscious ability of the subjects to incorporate the behavior of the haptic cue into their stepping behavior; Tapping at $2 \times \boldsymbol{\omega}_{\text {goal }}$ tries to take advantage of the astute ability of humans to synchronize [31]; Fast Tapping and Vibration both serve as haptic versions of an alarm, turning on to quickly direct the subject's attention toward a problem.

\section{EXPERIMENT PROTOCOL}

We cleared the experiment with the IRB and recruited subjects with flyers posted on campus. Fourteen female and twenty-two male subjects between the ages of 18-30 were recruited. Each subject was paid \$20 cash for participating. All subjects completed an informed consent form and a health survey to verify their ability to safely participate. A sheet was hung around the stair climber so as to block any visual distractions for the subject, and the subject wore industrial earphones playing pink noise to block any audible distractions or stepping cues (such as the noise of the motor). Subjects were provided a bottle of water and a fan to keep cool.

Subjects were instructed to step on the stair climber as consistently as possible at a fast workout pace. This was done for a 30 -second warm-up period followed by a 30second baseline period, as shown in Fig. 4. The video game then appeared onscreen which the subject played while continuing to step at the same consistent pace for one more minute. We instructed the subjects that performance on the stepping task and video game were equally important. During half of the time the subject was playing the video game, one of the five haptic cues was provided. Each haptic cue was explained to the subject before it was used. Whether the cue was provided during the first half or second half of the video game was randomized, and the order in which the haptic cues were presented to each subject was also randomized. The goal speed of the haptic cue, $\omega_{\text {goal }}$, was set at the subject's average speed of the first minute. Thus, the haptic cue attempted to keep the subject stepping at the same pace he or she had been stepping during the first minute. At the end of this second minute, the trial stopped and the subject was allowed to relax until rested. Additional trials were conducted for each of the four remaining haptic cues. Computer data was recorded during each trial, and the subject also completed a questionnaire about each trial.

This protocol allows us to quantitatively find whether or not the haptic cue improved performance on the physical task (stepping consistently) without deteriorating performance on the mental task (playing the video game). 
TABLE I

TRIAL VALIDITY AND CUE DETECTION

\begin{tabular}{lccccc} 
& Vibration & $\begin{array}{c}\text { Tapping } \\
\text { at } 2 \times \boldsymbol{\omega}_{\text {goal }}\end{array}$ & E-Boost & $\begin{array}{c}\text { Asymmetric } \\
\text { Resistance }\end{array}$ & $\begin{array}{c}\text { Fast } \\
\text { Tapping }\end{array}$ \\
\hline Cue on, cue felt & 14 & 35 & 10 & 9 & 10 \\
Cue on, cue not felt & 5 & 1 & 23 & 4 & 2 \\
Cue not on, cue felt & 2 & 0 & 1 & 5 & 2 \\
Cue not on, cue not felt & 15 & 0 & 2 & 5 & 5 \\
Invalid trial & 0 & 0 & 0 & 13 & 17 \\
\hline Total & 36 & 36 & 36 & 36 & 36
\end{tabular}

\section{RESUlTs}

\section{A. Trial validity and cue detection}

During our pilot studies, almost all subjects slowed down when the video game started. During the experiment, however, not all subjects slowed down; some subjects were able to maintain a consistent speed and some actually sped up when the video game started. Thus, in these trials, the haptic cue never turned on. Obviously, these trials where the haptic cues were never encountered are not very informative for our study. They inform us how often subjects slowed down, but we are not interested in this. Rather, we are interested in whether or not the haptic cues can assist a subject when he slows down. Also, due to two errors in the Asymmetric Resistance and Fast Tapping programs, a series of these trials did not function correctly and therefore were thrown out. A breakdown of trial validity and haptic cue detection is given in Table I.

\section{B. Quantitative data results}

The primary question to be answered by the quantitative data is how consistently subjects kept their speed. The speed of each subject was recorded throughout all four periods (warm-up, baseline, game_no_cue, game_with_cue) of each trial. We calculated the velocity mean and standard deviation of each period. Then we subtracted the baseline values of these metrics from the game_no_cue and game_with_cue values.

$$
\begin{aligned}
& \Delta \bar{\mu}_{\text {vel_cue_on }}=\bar{\mu}_{\text {vel_cue_on }}-\bar{\mu}_{\text {vel_baseline }} \\
& \Delta \bar{\sigma}_{\text {vel_cue_on }}=\bar{\sigma}_{\text {vel_cue_on }}-\bar{\sigma}_{\text {vel_baseline }} \\
& \Delta \bar{\mu}_{v e l_{\text {_cue_off }}}=\bar{\mu}_{\text {vel_cue_off }}-\bar{\mu}_{\text {vel_baseline }} \\
& \Delta \bar{\sigma}_{\text {vel_cue_off }}=\bar{\sigma}_{\text {vel_cue_off }}-\bar{\sigma}_{\text {vel_baseline }}
\end{aligned}
$$

These $\Delta$ values were then averaged for all trials by cue type. The results are shown graphically ${ }^{1}$ in Figs. 5 and 6. We performed matched-pairs t-tests on the $\Delta$ metrics for each cue to determine if there was a statistically significant difference between the values for cue_on and cue_off. Those pairs that had $\mathrm{p}<0.05$ are marked with a $\sqrt{ }$ in Figs. 5 and 6 and those that did not are marked with $\mathrm{a} \times$.

These metrics provide a measure of how much the subject sped up or slowed down when playing the video game $(\Delta \mu)$ and how much their velocity variation changed $(\Delta \sigma)$. However, the E-Boost cue cannot be appropriately analyzed using velocity because it directly changes the user's velocity. Therefore, we calculated $\Delta$ metrics on user power input as well, which provide an unbiased metric of user performance. These power metrics for mean and standard deviation are shown in Figs. 7 and 8, respectively.

\footnotetext{
${ }^{1}$ The numerical values for these graphs are included in the thesis version of this paper [29].
}

Performance on the video game mental distraction task is shown in Fig. 9.

\section{Qualitative results}

Subjects completed questionnaires after each trial that included evaluation of their performance and the haptic cue. The answers were analyzed using a Kruskal-Wallis parametric ANOVA with haptic cue as the factor. Most results did not produce statistically significantly different results for the various haptic cues, but some did. The results for three of the questions are shown in Fig. 10. The questions are:

- "Did you feel the cue?" ( $p=0.00)$ The results for this question show the percent of subjects who reported feeling the cue during the trials when the cue actually came on.

- "Was the cue helpful?" ( $\mathrm{p}=0.048)$ The results shown for this question only include those subjects who felt the cue when it actually came on.

- "Was the cue annoying?" ( $p=0.109)$ These results also only include those subjects who felt the cue when it actually came on.

Results for all other questions are included in the thesis version of this paper [29].

\section{DISCUSSION}

Vibration: This cue was not noticed $26 \%$ of the time it was on and was reported as annoying by $43 \%$ of subjects who felt it. This is consistent with previous research [32] that suggests users need to be able to set the intensity of vibration so as to avoid these two problems. The cue was somewhat effective in keeping subjects' mean velocity and power above $\omega_{\text {goal }}$, but did not statistically significantly reduce the amount of variance in stepping speed and power. Overall, the low reliability of subject detection of this cue indicates that vibration may be a poor means of haptic communication during active machine interaction. Additionally, most manual control tasks encounter enough physical noise to risk masking a vibration communication.

Tapping at $2 \times \omega_{\text {goal }}$ : This cue was reported as too difficult to follow by many subjects. It did, however, keep the subjects stepping at a speed slightly above $\boldsymbol{\omega}_{\text {goal }}$, although it had a detrimental impact on speed variance. It was also the only cue to have a statistically significant detrimental impact on the mental task.

E-Boost: This cue was preferred by some subjects, perhaps because it was hardly noticeable, being noticed by only $30 \%$ of subjects. Fewer subjects reported this cue as annoying, confusing, or distracting than any other cue. However, looking at the power metrics, it can be seen that this cue reduced the total amount of power subjects were expending, obviously counter-productive to the task of exercising. If the task was something other than exercise, however, this cue may be preferred because it minimized velocity standard deviation more than any other cue.

Asymmetric Resistance: This cue was only noticed by $70 \%$ of subjects. However, most users reported feeling an overall increase in the resistance encountered on both legs, not an asymmetry in resistance as was intended. This was likely due to the virtual flywheel's inertia masking the 


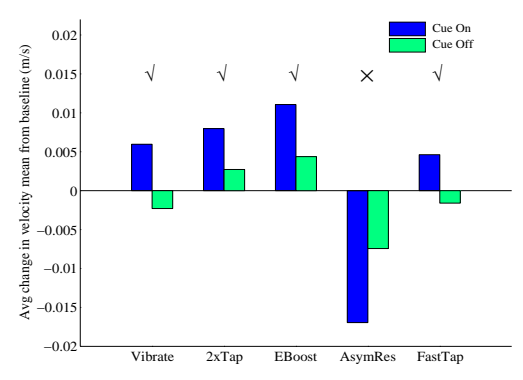

Fig. 5. Change in velocity mean $(\mathrm{m} / \mathrm{s})$

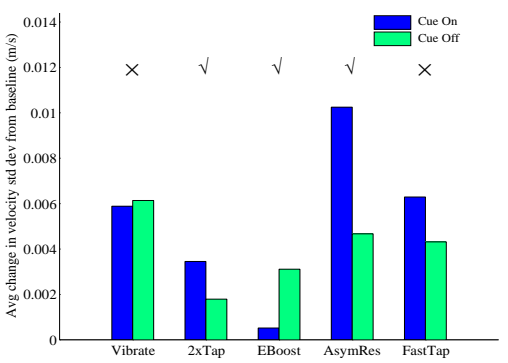

Fig. 6. Change in velocity std dev $(\mathrm{m} / \mathrm{s})$

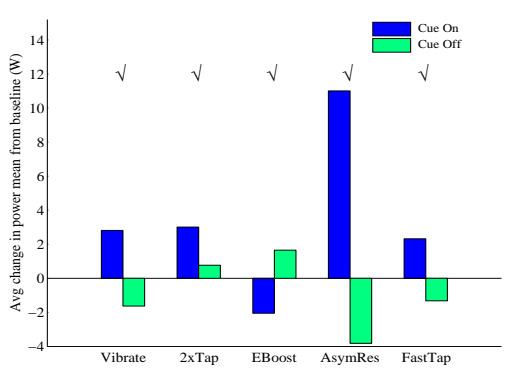

Fig. 7. Change in power mean (W)

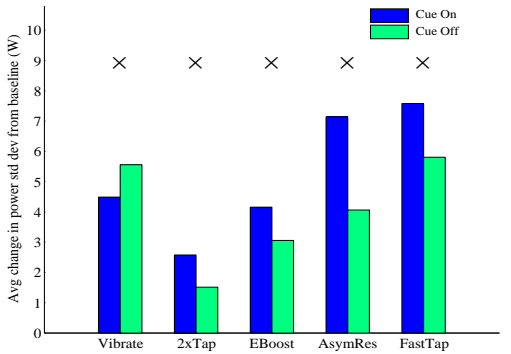

Fig. 8. Change in power std dev (W)

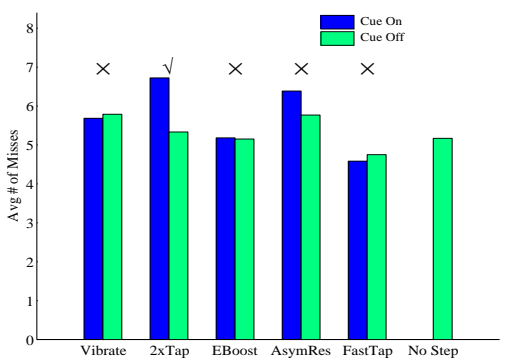

Fig. 9. Video game performance (Misses)

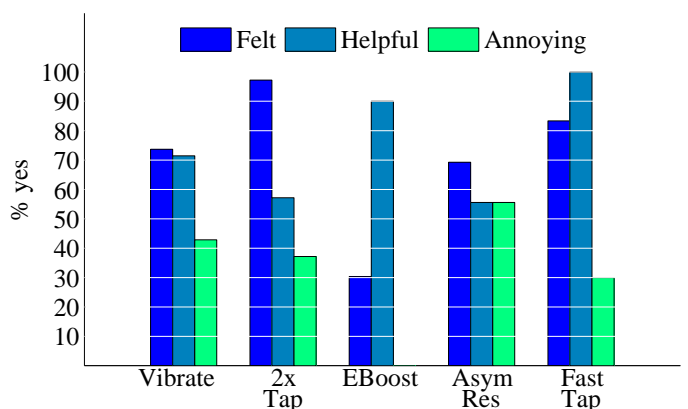

Fig. 10. Questionnaire results

"Did you feel the cue?" ( $\mathrm{p}=0.00)$

"Was the cue helpful?" $\quad(p=0.048)$

"Was the cue annoying?" $(\mathrm{p}=0.109)$

instantaneous change in damping. It could be that due to this flaw, subjects had a difficult time detecting the cue because they had to detect a change in the resistance in time instead of in space. That is, they had to decide whether the resistance being felt at a given time was different from the resistance felt a few seconds prior. This is likely much more difficult than comparing the resistance felt on each leg. Perhaps the lesson to be learned from this is to either have haptic communications that are easily distinguishable as on or off, or have a comparison between two feelings in space, but do not require subjects to make comparisons in time.

Several subjects got "stuck" going slow when this haptic cue increased resistance so much that they could not overcome the additional resistance. This again shows us that users must have control of haptic communication intensity.

Fast Tapping: This cue was the preferred cue based on the subjective questionnaire results and was also listed by multiple subjects as their favorite cue. The quantitative data shows that it succeeded in keeping up both subject speed and power, although not to the same magnitude that the other cues did.

\section{CONCLUSION}

The results of our experiment show that haptic communications can be used successfully to modify the behavior of a individual in a manual control task, even in the presence of a mental distraction. Specifically, they show that haptic cues can help an individual maintain a more consistent level of exercise intensity when using an exercise machine.

Results of this experiment provide new quantitative and qualitative insight into the design of haptic communications. The fact that users found the E-Boost cue to be the least confusing cue lends credit to the idea that haptic cues that mimic a desired behavior are easily understood by users. Both E-Boost and Fast Tapping were subjectively preferred by subjects, even though they were very different cues in that E-Boost was very subtle and Fast Tapping was very noticable. Thus, perhaps users should be able to select their preferred haptic communication. However, doing so would make standardization of haptic signals difficult. The result that some subjects could not detect the intensity level of our Vibration and Asymmetric Resistance cues while other subjects were annoyed or encountered problems due to the 
intensity level supports previous claims that subjects should have control of the intensity of haptic communications. Tapping at $2 \times \omega_{\text {goal }}$ was the only cue in this experiment that was always on, and subjects reported feeling less "in control" of the machine during this cue than during any other cue. Therefore, perhaps a haptic sensation that is always present may decrease the user's sense of feeling in control. Other forms of continuous haptic communications should be investigated.

The thesis from which this paper is drawn is available online [29]. An extended list of relevant resources can be found at http://lims.mech.northwestern.edu/projects/stairclimber/

The authors would like to acknowledge the National Science Foundation grant ECS 0433948 for support of this work.

\section{REFERENCES}

[1] A. Chan, K. MacLean, and J. McGrenere, "Learning and identifying haptic icons under workload," in First Joint Eurohaptics Conference and Symposium on Haptic Interfaces for Virtual Environment and Teleoperator Systems, 18 March 2005, pp. 432-439.

[2] H. S. Vitense, J. A. Jacko, and V. K. Emery, "Multimodal feedback: An assessment of performance and mental workload," Ergonomics, vol. 46 , no. $1-3$, pp. $68-87,2003$.

[3] C. Basdogan, C.-H. Ho, M. A. Srinivasan, and M. Slater, "An experimental study on the role of touch in shared virtual environments," ACM Human Computer Interactions, vol. 7, no. 4, pp. 440-463, 2000.

[4] J. C. Roberts and K. Franklin, "Haptic glyphs (hlyphs) - structured haptic objects for haptic visualization," in First Joint Eurohaptics Conference and Symposium on Haptic Interfaces for Virtual Environment and Teleoperator Systems, March 18-20 2005, pp. 369-374.

[5] S. A. Wall and W. S. Harwin, "Quantification of the effects of haptic feedback during a motor skills task in a simulated environment," in 2nd PHANToM Users Research Symposium, 2000.

[6] A. F. Rovers and H. A. van Essen, "Guidelines for haptic interpersonal communication applications: An exploration of foot interaction styles," Virtual Reality, vol. 9, pp. 177-191, 2006.

[7] I. Oakley, S. Brewster, and P. Gray, "Can you feel the force? An investigation of haptic collaboration in shared editors," in Eurohaptics, 2001 .

[8] M. K. O’Malley, A. Gupta, M. Gen, and Y. Li, "Shared control in haptic systems for performance enhancement and training," Journal of Dynamic Systems, Measurement, and Control, vol. 128, pp. 75-85, March 2006.

[9] E. Paulos, "Connexus: A communal interface," in Conference on Designing for User Experiences, San Francisco, CA, 2003, pp. 1-4.

[10] L. Rovers and H. van Essen, "Design and evaluation of hapticons for enriched instant messaging," Virtual Reality, vol. 9, pp. 177-191, 2004.

[11] A. F. Rovers and H. A. van Essen, "HIM: A framework for haptic instant messaging," in CHI 2004, Vienna, Austria, April 24-29 2004, pp. 1313-1316.

[12] A. Tang, P. McLachlan, K. Lowe, C. R. Saka, and K. MacLean, "Perceiving ordinal data haptically under workload," in 7th International Conference on Multimodal Interfaces, ACM. New York, NY: ACM Press, 2005, pp. 317-324.
[13] L. M. Brown, S. A. Brewster, and H. C. Purchase, "A first investigation into the effectiveness of tactons," in First Joint Eurohaptics Conference and Symposium on Haptic Interfaces for Virtual Environment and Teleoperator Systems, 2005, pp. 167-176.

[14] A. Chang, S. O'Modhrain, R. Jacob, E. Gunther, and H. Ishii, "ComTouch: Design of a vibrotactile communication device," in Conference on Designing Interactive Systems, 2002, pp. 312-320.

[15] J. B. F. van Erp and H. A. H. C. van Veen, "Vibro-tactile information presentation in automobiles," Eurohaptics, 2001.

[16] T. A. Dingus, D. V. McGehee, N. Manakkal, S. K. Jahns, C. Carney, and J. M. Hankey, "Human factors field evaluation of automotive headway maintenance/collision warning devices," Human Factors vol. 39, no. 2, pp. 216-229, 1997.

[17] M. Enriquez, O. Afonin, B. Yager, and K. Maclean, "A pneumatic tactile alerting system for the driving environment," in Proceedings of the 2001 workshop on perceptive user interfaces, 2001, pp. 1-7.

[18] C. Ho, H. Z. Tan, and C. Spence, "Using spatial vibrotactile cues to direct visual attention in driving scenes," Science Direct, vol. Transportation Research Part F 8, pp. 397-412, 2005.

[19] P. Griffiths and R. B. Gillespie, "Shared control between human and machine: Haptic display of automation during manual control of vehicle heading," in 12th International Symposium on Haptic Interfaces for Virtual Environment and Teleoperator Systems (HAPTICS '04), March 27-28 2004, pp. 358-366.

[20] M. Steele and R. B. Gillespie, "Shared control between human and machine: Using a haptic steering wheel to aid in land vehicle guidance," in Human Factors and Ergonomics Society 45th Annual Meeting, Minneapolis/St. Paul, MN, Oct 8-12 2001, pp. 1671-1675.

[21] D. A. Abbink, F. C. T. van der Helm, and E. R. Boer, "Admittance measurements of the foot during 'maintain position' and 'relax' tasks on a gas pedal," in IEEE International Conference on Systems, Man, and Cybernetics, 2004, pp. 2519-2524.

[22] M. Enriquez and K. E. MacLean, "Impact of haptic warning signal reliability in a time-and-safety-critical task," in 12th International Symposium on Haptic Interfaces for Virtual Environment and Teleoperator Systems (HAPTICS '04), March 27-28 2004, pp. 407-414.

[23] Cateye GameBike. [Online]. Available: http://www.cateyefitness.com/GameBike/index.html

[24] InterAction Labs. [Online]. Available: http://www.powergridfitness.com/

[25] G. Wijnalda, S. Pauws, F. Vibnoli, and H. Stuckenschmidt, "A personalized music system for motivation in sport performance," Pervasive Computing, pp. 26-32, July-September 2005.

[26] InCorp Ventures. [Online]. Available: http://www.mytrakhealth.com/

[27] D-Squared Technologies. [Online]. Available: http://www.tvpedaler.com

[28] The Entertrainer. [Online]. Available: http://www.theentertrainer.com/

[29] A. R. Ferber, "Affecting exercise intensity through haptic communications," Master's thesis, Northwestern University, Evanston, IL, June 2007.

[30] A. M. Simon, R. B. Gillespie, and D. P. Ferris, "Symmetry-based resistance as a novel means of lower limb rehabilitation," Journal of Biomechanics, vol. 40, no. 6, pp. 1286-1292, July 142007.

[31] N. P. Lago and F. Kon, "The quest for low latency," in International Computer Music Conference, 2004, pp. 33-36, Department of Computer Science University of São Paulo.

[32] J. B. F. van Erp, "Guidelines for the use of vibro-tactile displays in human computer interaction," in Eurohaptics, 2002. 